\title{
Curva Nacional da Escala Motora Infantil de Alberta e Escores de Lactentes Expostos ao HIV
}

National Curve of the Alberta Infant Motor Scale and Scores of Infants Exposed to HIV

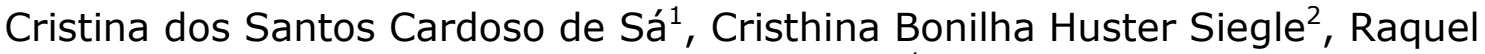
de Paula Carvalho ${ }^{1}$

1.Fisioterapeuta, Doutora, Docente associada do Curso de Fisioterapia, Universidade Federal de São Paulo, Santos-SP, Brasil.

2.Fisioterapeuta, Residente do Programa de Residência Multidisciplinar, Universidade de São Paulo, Santos-SP, Brasil.

\section{Resumo}

Objetivo. Comparar se o padrão de desenvolvimento neuromotor de lactentes expostos ao HIV é diferente do padrão de desenvolvimento de lactentes não expostos, e estabelecer a curva de referência do desenvolvimento motor para lactentes expostos ao HIV. Método. Cento e setenta e três lactentes expostos ao HIV nas idades de recém-nascido a 18 meses foram avaliados mensalmente por meio da escala brasileira motora infantil de Alberta e os escores de desempenho motor foram comparados à referência de lactentes brasileiros. Resultados. Os escores de desempenho motor foram similares, e nas idades de 0 a 3 meses e de 15 a 18 meses os pontos de corte dos escores para cada percentil são os mesmos. Conclusão. Lactentes expostos ao HIV possuem padrão de desenvolvimento neuromotor similar ao padrão de desenvolvimento neuromotor de lactentes não expostos ao HIV.

Unitermos. Desenvolvimento infantil; lactente; HIV/AIDS

\begin{abstract}
Objective. To compare the pattern of neuromotor development of infants exposed to HIV is different from the pattern of development of non-exposed infants and to establish the motor development reference curve for infants exposed to HIV. Method. one hundred and seventy-three HIV-exposed infants at ages 0-18 months were evaluated by means of Alberta Infant Motor Scale, Brazilian version, monthly, and motor performance scores were compared to Brazilian infants. Results. The motor performance scores were similar, and in ages from 0 to 3 months and from 15 to 18 months the cut off points of performance score for each percentile are the same. Conclusion. Infants exposed to HIV have standard neuromotor development similar to neuromotor development pattern of unexposed infants to HIV.
\end{abstract}

Keywords. Child Development; infant; HIV/AIDS

Trabalho realizado Universidade Federal de São Paulo, UNIFESP, Santos-SP, Brasil.

Conflito de interesse: não Recebido em: $2018 \quad$ Aceito em: 2018

Endereço para correspondência: Cristina SC de Sá. R. Silva Jardim, 136. Departamento de Ciências do Movimento Humano. Fone: +55(13)3878-3700. Email: cristina.sa@uol.com.br 


\section{INTRODUÇÃO}

O desenvolvimento motor é caracterizado por um processo em que ocorrem modificações no comportamento, incluindo mudanças na postura e no movimento, as quais estão relacionadas à idade do indivíduo ${ }^{1,2}$. Os primeiros anos de vida são considerados extremamente importantes para a aquisição e aperfeiçoamento das primeiras formas do movimento. Durante a primeira infância, que compreende a idade de zero a seis anos, ocorre maior plasticidade e mudanças neuropsicomotoras, que contribuem para o melhor desenvolvimento da criança e um ritmo de desenvolvimento rápido ${ }^{3,4}$.

O desenvolvimento neuromotor ocorre ao longo da evolução cronológica de modo contínuo, e resulta da interação entre fatores biológicos, sociais, econômicos e ambientais $^{5-7}$. Existem fatores facilitadores ao desenvolvimento e fatores de risco que aumentam a chance de aparecimento de anormalidades neuropsicomotoras. Pode-se citar como fatores de risco doenças infecciosas, infecções congênitas, lesões cerebrais, asfixia perinatal, baixo peso ao nascer, idade gestacional, idade materna, tempo de permanência no berçário, tempo passado com os pais, estímulos no lar, baixa escolaridade materna, baixa condição socioeconômica entre outros ${ }^{7-18}$. Um dos fatores biológicos que pode afetar o desenvolvimento é a infecção pelo vírus HIV, que tem afinidade pelo sistema nervoso central (SNC) $)^{8,9,19}$. Não somente a infecção, mas também a exposição do feto ao vírus, associada à terapia 
antirretroviral empregada para prevenir a transmissão vertical, podem comprometer o desenvolvimento desse lactente $e^{9,20-23}$. Portanto, a identificação precoce de desvios do desenvolvimento é prioritária para diminuir os efeitos do risco e potencializar o desenvolvimento desses lactentes.

Os primeiros sintomas de alterações do desenvolvimento são percebidos por meio de atrasos motores que podem ser detectados quando aplicadas escalas apropriadas para avaliação do desenvolvimento ${ }^{24,25}$. A Alberta Infant Motor Scale (AIMS), Escala Motora Infantil de Alberta, vem sendo utilizada no monitoramento do desenvolvimento neuromotor e detecção de atrasos e anormalidades do desenvolvimento. No entanto, tem-se utilizado a AIMS, já validada para a população pediátrica brasileira ${ }^{26}$, nos estudos com lactentes expostos ao HIV ${ }^{27}$. Portanto, há necessidade de verificar se o padrão dos lactentes expostos ao HIV é similar ou não ao padrão dos lactentes não expostos ao HIV.

Este estudo objetivou comparar o padrão de desenvolvimento neuromotor de lactentes expostos ao HIV ao padrão de desenvolvimento neuromotor de lactentes não expostos ao HIV e estabelecer a curva de referência do desenvolvimento motor para lactentes expostos ao HIV.

\section{MÉTODO}

\section{Participantes}

Participaram deste estudo 173 lactentes expostos ao HIV, de ambos os gêneros, com idade de recém-nascido 
(RN) a 18 meses. Esses lactentes estavam em atendimento no SENIC/SECRAIDS (Seção Núcleo Integrado de Atendimento à Criança/Seção Centro de Referência em AIDS) de Santos-SP.

A amostra deste estudo refere-se a 95\% dos lactentes expostos ao HIV atendidos neste centro de referência no período de agosto de 2010 a dezembro de 2015.

A Tabela 1 mostra as características dos bebês avaliados por meses de idade, em termos de gênero, uso da terapia antirretroviral (TARV) pela gestante durante a gestação e durante o momento do parto, número de consultas pré-natais realizadas, tipo de parto e baixo peso ao nascer. Os lactentes prematuros foram excluídos do estudo, pois a prematuridade já é um fator de risco para o desenvolvimento. Assim como lactentes que apresentaram má formação, síndromes genéticas, alterações congênitas, deformidades posturais ou paralisia cerebral.

Foram realizadas 822 avaliações, sendo 386 realizadas em meninas e $436 \mathrm{em}$ meninos. Alguns lactentes foram avaliados em mais de uma idade, por isso o número de avaliações é maior do que o número de participantes.

Este estudo foi aprovado pela comissão de ética em Pesquisa da instituição sede sob o $n^{0}$ 0600/11.

\section{Procedimento}

Os pais dos lactentes expostos ao HIV em acompanhamento mensal pela equipe médica do SENIC/CRAIDS Santos foram informados e convidados a 
Tabela 1. Caracterização da amostra

\begin{tabular}{|c|c|c|c|c|c|c|c|c|c|c|c|c|c|c|c|}
\hline \multirow{2}{*}{$\begin{array}{l}\text { Idade } \\
\text { Meses } \\
\end{array}$} & \multicolumn{2}{|c|}{$\begin{array}{l}\text { Gênero } \\
\text { (n) }\end{array}$} & \multicolumn{3}{|c|}{$\begin{array}{l}\text { Uso de TARV } \\
\text { durante a } \\
\text { gestação }(n)\end{array}$} & \multicolumn{3}{|c|}{$\begin{array}{l}\text { Uso de AZT injetável } \\
\text { no momento do parto } \\
\text { (n) }\end{array}$} & \multicolumn{3}{|c|}{$\begin{array}{c}\text { Número } \\
\text { consultas pré- } \\
\text { natal }(\mathbf{n})\end{array}$} & \multicolumn{2}{|c|}{$\begin{array}{l}\text { Tipo de } \\
\text { parto }(n)\end{array}$} & \multicolumn{2}{|c|}{ Peso ao nascimento } \\
\hline & $\mathbf{F}$ & M & $\mathbf{S}$ & $\mathbf{N}$ & SR & $\mathbf{S}$ & $\mathbf{N}$ & SR & $<6$ & $>6$ & SR & $\mathbf{N a}$ & C & $<2500 \mathrm{~g}$ & $>2500 \mathrm{~g}$ \\
\hline RN & 18 & 17 & 27 & 5 & 3 & 29 & 3 & 3 & 10 & 16 & 9 & 13 & 22 & 7 & 28 \\
\hline 1 & 17 & 27 & 32 & 7 & 5 & 36 & 5 & 3 & 14 & 20 & 10 & 18 & 26 & 9 & 35 \\
\hline 2 & 28 & 26 & 42 & 10 & 2 & 43 & 10 & 1 & 17 & 23 & 14 & 19 & 35 & 10 & 44 \\
\hline 3 & 31 & 26 & 43 & 7 & 7 & 43 & 10 & 4 & 17 & 26 & 14 & 23 & 34 & 14 & 43 \\
\hline 4 & 27 & 28 & 45 & 9 & 1 & 46 & 8 & 1 & 22 & 20 & 13 & 15 & 40 & 14 & 41 \\
\hline 5 & 20 & 26 & 36 & 9 & 1 & 37 & 8 & 1 & 14 & 20 & 12 & 15 & 31 & 11 & 35 \\
\hline 6 & 17 & 22 & 28 & 9 & 2 & 29 & 7 & 3 & 16 & 15 & 8 & 15 & 24 & 8 & 31 \\
\hline 7 & 19 & 23 & 34 & 8 & 0 & 38 & 4 & 0 & 10 & 21 & 11 & 13 & 29 & 10 & 32 \\
\hline 8 & 21 & 26 & 34 & 12 & 1 & 40 & 6 & 1 & 15 & 21 & 11 & 17 & 30 & 11 & 36 \\
\hline 9 & 25 & 24 & 37 & 11 & 1 & 44 & 4 & 1 & 17 & 17 & 15 & 17 & 32 & 12 & 37 \\
\hline 10 & 16 & 23 & 31 & 7 & 1 & 33 & 5 & 1 & 10 & 20 & 9 & 16 & 23 & 10 & 29 \\
\hline 11 & 17 & 27 & 34 & 8 & 2 & 37 & 6 & 1 & 12 & 19 & 13 & 17 & 27 & 11 & 33 \\
\hline 12 & 23 & 22 & 40 & 5 & 0 & 39 & 5 & 1 & 11 & 20 & 14 & 14 & 31 & 12 & 33 \\
\hline 13 & 16 & 21 & 27 & 7 & 3 & 28 & 8 & 1 & 7 & 20 & 10 & 15 & 22 & 8 & 29 \\
\hline 14 & 17 & 24 & 35 & 6 & 0 & 35 & 6 & 0 & 10 & 17 & 14 & 21 & 20 & 10 & 31 \\
\hline 15 & 21 & 23 & 31 & 10 & 3 & 39 & 4 & 1 & 14 & 21 & 9 & 10 & 34 & 8 & 36 \\
\hline 16 & 19 & 10 & 23 & 5 & 1 & 27 & 2 & 0 & 7 & 13 & 9 & 11 & 18 & 8 & 21 \\
\hline 17 & 18 & 23 & 32 & 8 & 1 & 36 & 4 & 1 & 12 & 18 & 11 & 16 & 25 & 10 & 31 \\
\hline 18 & 16 & 18 & 27 & 6 & 1 & 30 & 3 & 1 & 4 & 15 & 15 & 9 & 25 & 7 & 27 \\
\hline
\end{tabular}

$\mathrm{n}=$ número de crianças avaliadas determinado mês; $\mathrm{F}=$ feminino; $\mathrm{M}=$ Masculino; $\mathrm{S}=$ sim; $\mathrm{N}=$ não; $\mathrm{SR}=$ sem registro; $\mathrm{Na}=$ natural; $\mathrm{C}=$ cesárea; $\mathrm{RN}=$ recém-nascido.

participar com seus filhos do estudo para avaliação do desenvolvimento neuromotor. Nesse equipamento da Secretaria Municipal de Saúde, a equipe é formada por pediatra, fisioterapia, psicólogo, enfermeira, auxiliar de enfermagem e assistente social. Após a autorização dos pais e/ou responsáveis pela assinatura do termo de consentimento livre esclarecido, os lactentes foram avaliados em uma sala em condições ideais de iluminação, temperatura e ventilação.

Para avaliar o desenvolvimento motor dessas crianças, utilizou-se a versão brasileira da Alberta Infant Motor Scale, 
Escala Motora Infantil de Alberta, que foi validada em 2012 por Saccani e Valentin ${ }^{26}$. A escala é composta por 58 critérios motores, distribuídos nas quatro subescalas (posição: prona, supina, sentada e em pé), que avaliam os padrões motores e posturas usando-se os critérios de alinhamento, equilíbrio postural e controle da musculatura em movimentos antigravitacionais, de crianças de RN a 18 meses $^{26,28}$.

Em um colchonete e com brinquedos adequados a idade, o lactente foi colocado nas posições e observou-se as atividades realizadas em cada posição. O lactente recebia pontuação " 1 " ou " 0 " de acordo com o que foi observado ou não observado, respectivamente. A maior pontuação possível da posição prona é 21 , supina 9 , sentada 12 e em pé 16. O escore bruto foi obtido a partir da soma do escore em cada uma das subescalas e foi convertido em um percentil. Percentis foram agrupados em categorias de desenvolvimento motor: abaixo de $5 \%$, considerou-se que o lactente apresenta desempenho motor anormal; entre $5 \%$ e $25 \%$, desempenho motor suspeito; e acima de $25 \%$, desempenho motor normal ${ }^{26}$. A classificação de percentil é normatizada para permitir a comparação entre o lactente e um grupo de referência, podendo, assim, haver a observação de deficiência nas aquisições motoras que o lactente deveria possuir ${ }^{28}$. Por meio destas posições é possível observar a maturação do sistema nervoso central (SNC), a dinâmica motora e a sequência em que o desenvolvimento motor está acontecendo. 
As avaliações aconteciam mensalmente, mas nem todos os lactentes foram avaliados em todas as idades, de RN a 18 meses. Por meio dos prontuários de cada lactente foram obtidos dados da história do lactente, assim como o ano de início de tratamento da mãe, o número de consultas pré-natal, o tipo de parto e se a gestante fez o uso de medicamento durante gestação e parto.

\section{Análise estatística}

Os dados foram descritos por meio de média, desvio-padrão, mínimo e máximo. Os testes foram realizados usando o Programa $\mathrm{R}$ e foi considerado nível de significância de 5\%. Para comparar o escore bruto total do desempenho motor de lactentes expostos ao HIV com os dados de referência da população brasileira, foi aplicado o teste t one sample.

Para estimar os pontos de corte do escore bruto dos lactentes expostos ao HIV para os percentis de interesse, foi aplicado o modelo de regressão quantílica segundo Koenker e Basset ${ }^{29}$. A partir dos pontos de corte estimados, foi criada a curva de referência para os lactentes expostos ao HIV.

\section{RESULTADOS}

A Tabela 2 mostra a comparação das médias de escores brutos obtidos a cada mês para os lactentes expostos ao HIV com o valor referência dos lactentes brasileiros $^{26}$. Os comportamentos motores foram 
Tabela 2. Média e desvio-padrão dos escores brutos da AIMS para a população exposta ao HIV e dados normativos da versão brasileira da AIMS em relação à idade.

\begin{tabular}{|c|c|c|c|c|c|c|}
\hline \multirow{2}{*}{$\begin{array}{c}\text { Idade } \\
\text { (meses) }\end{array}$} & \multicolumn{2}{|c|}{ lactentes não expostos } & \multicolumn{2}{|c|}{ Dados normatizados - AIMS } & \multirow[t]{2}{*}{ Teste $\mathrm{t}$} & \multirow[t]{2}{*}{$p$} \\
\hline & Média \pm DP & Min-Máx & Média \pm DP & Min - Máx & & \\
\hline $\mathbf{R N}$ & $4,33 \pm 1,34$ & $3-8$ & $4,53 \pm 1,48$ & $3-7$ & 0,126 & 0,9 \\
\hline 1 & $6,2 \pm 1,3$ & $4-9$ & $6,95 \pm 1,56$ & $3-13$ & 4,492 & $0,0001^{*}$ \\
\hline 2 & $8,43 \pm 1,91$ & $6-13$ & $8,89 \pm 2,13$ & $5-20$ & $-0,202$ & 0,840 \\
\hline 3 & $11,2 \pm 2,93$ & $6-16$ & $12,61 \pm 2,15$ & $8-17$ & 4,980 & $0,0001^{*}$ \\
\hline 4 & $14,9 \pm 3,5$ & $8-23$ & $15,74 \pm 3,37$ & $8-23$ & 0,928 & 0,356 \\
\hline 5 & $18 \pm 5,75$ & $7-29$ & $21,11 \pm 4,47$ & $14-33$ & 3,832 & $0,0001^{*}$ \\
\hline 6 & $22,6 \pm 6,38$ & $10-35$ & $27,10 \pm 4,85$ & $18-38$ & 3,926 & $0,0001^{*}$ \\
\hline 7 & $30,7 \pm 5,54$ & $18-42$ & $32,75 \pm 6,10$ & $15-46$ & 1,858 & 0,067 \\
\hline 8 & $36,8 \pm 7,66$ & $21-52$ & $38,51 \pm 6,98$ & $20-54$ & 1,88 & 0,062 \\
\hline 9 & $40,8 \pm 8,66$ & $21-52$ & $43,08 \pm 7,58$ & $28-53$ & 1,503 & 0,136 \\
\hline 10 & $43,4 \pm 8,15$ & $28-53$ & $48,05 \pm 6,47$ & $23-55$ & 1,912 & 0,59 \\
\hline 11 & $49,3 \pm 4,84$ & $35-58$ & $51,39 \pm 4,70$ & $35-58$ & 2,854 & $0,005^{*}$ \\
\hline 12 & $53,4 \pm 3,39$ & $47-58$ & $53,71 \pm 3,41$ & $40-58$ & $-0,913$ & 0,364 \\
\hline 13 & $54 \pm 3,92$ & $44-58$ & $55,49 \pm 2,58$ & $51-58$ & 1,048 & 0,298 \\
\hline 14 & $56,3 \pm 2,92$ & $46-58$ & $56,71 \pm 2,05$ & $51-58$ & 1,332 & 0,186 \\
\hline 15 & $56,9 \pm 2,00$ & $51-58$ & $57,41 \pm 1,19$ & $53-58$ & 1,112 & 0,269 \\
\hline 16 & $57,8 \pm 0,58$ & $55-58$ & $57,90 \pm 0,31$ & $57-58$ & 0,431 & 0,668 \\
\hline 17 & $57,8 \pm 0,88$ & $53-58$ & $57,68 \pm 1,13$ & $52-58$ & 0,068 & 0,946 \\
\hline 18 & $57,9 \pm 0,48$ & $56-58$ & $57,97 \pm 0,17$ & $57-58$ & 0,733 & 0,466 \\
\hline
\end{tabular}

semelhantes para RN e 20,40,70, 80, 90, 100, 120, 130, $14^{\circ}, 15^{\circ}, 16^{\circ}, 17^{\circ}$ e $18^{\circ}$ mês de idade. Nas idades de $1^{\circ}$, $3^{0}, 5^{0}, 6^{0}$ e $11^{0}$ mês, diferenças significativas foram observadas, com maior escore de desempenho motor dos lactentes expostos ao HIV.

A Tabela 3 indica os valores de referência estimados, ao longo da trajetória do desenvolvimento dos lactentes expostos ao HIV, do escore bruto para os pontos de corte de percentil 5\%, 10\%, 25\%, 50\%, 75\% e 90\%. Nota-se que nas extremidades da tabela, de RN a 3 meses e de 15 a 18 meses, os pontos de cortes são os mesmos para as idades, indicando baixo poder de diferenciação do escore de 
desenvolvimento neuromotor nessas idades entre lactentes expostos ao HIV com desenvolvimento motor típico e atípico.

Tabela 3. Valores de referência estimados da AIMS para performance motora de lactentes expostos ao HIV (escore bruto), por idade avaliada (em meses), para os pontos de corte de percentil $5 \%, 10 \%, 25 \%, 50 \%, 75 \%$ e $90 \%$.

\begin{tabular}{c|cccccc}
\hline Idade & $\mathbf{5 \%}$ & $\mathbf{1 0 \%}$ & $\mathbf{2 5 \%}$ & $\mathbf{5 0 \%}$ & $\mathbf{7 5 \%}$ & $\mathbf{9 0 \%}$ \\
\hline $\mathbf{R N}$ & 4 & 5 & 6 & 7 & 7,3 & 8 \\
$\mathbf{1}$ & 6 & 7 & 8 & 8 & 10 & 11 \\
$\mathbf{2}$ & 8 & 8,5 & 11 & 13 & 14 & 15 \\
$\mathbf{3}$ & 10 & 12 & 14 & 16 & 18 & 21 \\
$\mathbf{4}$ & 15 & 16 & 18 & 21 & 25 & 27 \\
$\mathbf{5}$ & 19,5 & 21 & 23 & 27 & 31 & 34 \\
$\mathbf{6}$ & 24 & 26 & 28 & 33 & 37 & 41 \\
$\mathbf{7}$ & 28 & 31 & 33 & 38 & 43,5 & 48 \\
$\mathbf{8}$ & 31 & 33 & 38 & 43,5 & 50 & 52 \\
$\mathbf{9}$ & 37 & 39 & 46 & 49,2 & 52 & 54 \\
$\mathbf{1 0}$ & 43 & 45 & 50,2 & 52 & 54 & 57 \\
$\mathbf{1 1}$ & 49 & 51 & 52 & 53 & 57 & 58 \\
$\mathbf{1 2}$ & 51 & 52 & 53 & 56 & 58 & 58 \\
$\mathbf{1 3}$ & 52,7 & 54 & 56 & 58 & 58 & 58 \\
$\mathbf{1 4}$ & 54,3 & 56 & 57 & 58 & 58 & 58 \\
$\mathbf{1 5}$ & 56 & 57 & 58 & 58 & 58 & 58 \\
$\mathbf{1 6}$ & 57 & 57,5 & 58 & 58 & 58 & 58 \\
$\mathbf{1 7}$ & 58 & 58 & 58 & 58 & 58 & 58 \\
$\mathbf{1 8}$ & 58 & 58 & 58 & 58 & 58 & 58 \\
\hline & & & 58 & & 58 & \\
\hline
\end{tabular}

Na Figura 1 observa-se a curva de referência, formada a partir dos valores da Tabela 3, para a amostra de lactentes expostos ao HIV. Assim, formam-se as curvas 
para cada percentil, de acordo com o escore bruto em cada mês. A partir do $15^{\circ}$ mês, a curva atinge um platô, assim como ocorre na curva da população de lactentes brasileiros, como visto na tabela 3 , que indica que a média para os escores a partir dos 15 meses é similar. A maior variabilidade pode ser observada entre o $3^{\circ}$ e o $15^{\circ}$ mês.

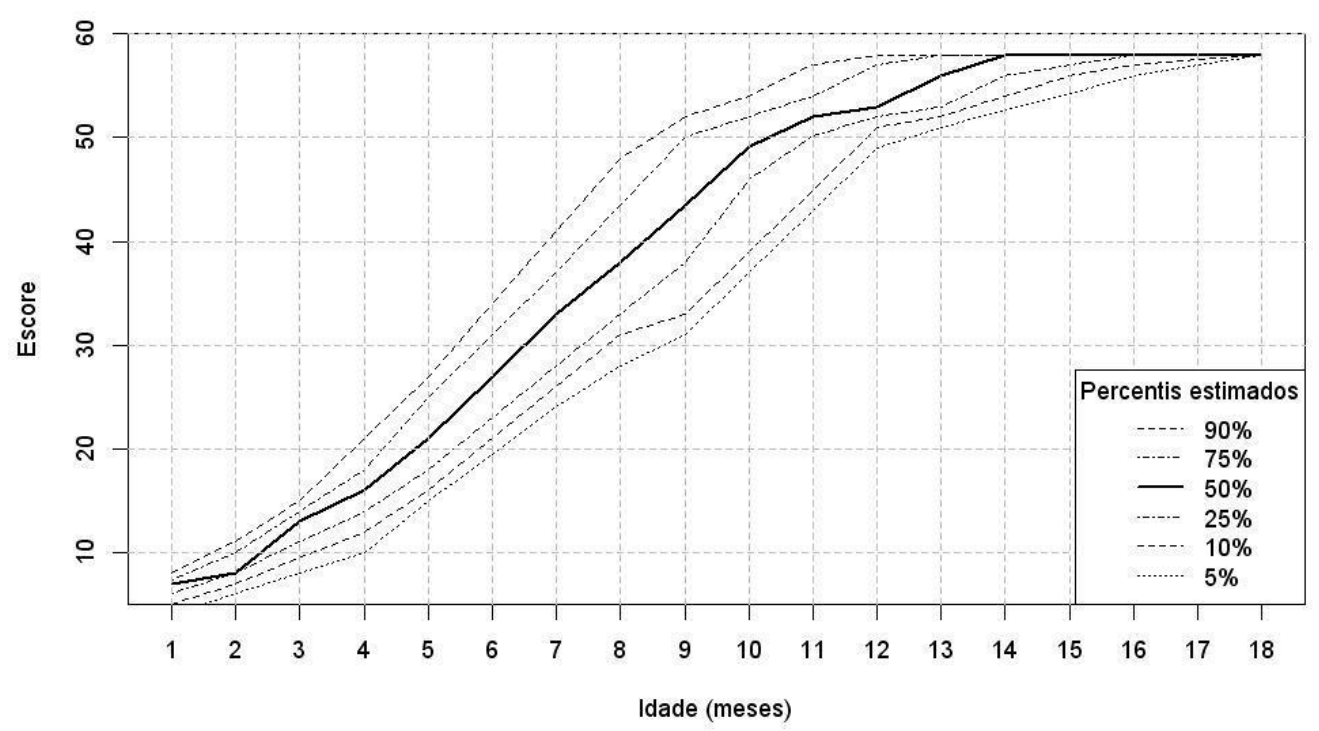

Figura 1. Curva da população pediátrica exposta ao HIV a partir dos percentis estimados para cada mês de acordo com o escore bruto obtido em cada idade dos lactentes expostos ao HIV

\section{DISCUSSÃO}

Esse estudo, ao comparar o desenvolvimento neuromotor de lactentes expostos ao HIV com a referência de lactentes brasileiros, observou que o padrão de desenvolvimento neuromotor de ambos os grupos foi similar, e ao estabelecer a curva de referência para os lactentes expostos ao HIV, percebe-se que esta é similar a 
curva percentílica do desenvolvimento para a população brasileira não exposta ao HIV. Sendo assim, a avaliação do desenvolvimento dos lactentes brasileiros expostos ao HIV pode ser avaliado com base na curva de referência brasileira para lactentes, sem prejuízo na sua avaliação.

$\mathrm{Na}$ comparação entre os escores brutos dos lactentes expostos ao HIV e os valores de referência para os lactentes brasileiros, pode-se afirmar que, no presente estudo, a maioria dos lactentes expostos ao HIV apresenta desenvolvimento neuromotor adequado para suas idades. Esses achados corroboram com os achados do estudo de Herrero et al. ${ }^{30}$ realizado na cidade de São Paulo, que avaliou o desenvolvimento neuromotor de lactentes expostos ao HIV por meio da AIMS e não encontrou atrasos no desenvolvimento motor em $90 \%$ dos lactentes. Sá et al. ${ }^{27}$ verificaram normalidade no desenvolvimento de lactentes expostos ao HIV avaliados pela AIMS. Entretanto, alguns lactentes apresentaram desenvolvimento suspeito, e outros, após 6 meses de idade, desenvolvimento anormal.

Os dados do presente estudo também indicaram que no $10,3^{\circ}, 5^{\circ}, 6^{\circ}$ e $11^{\circ}$ mês os lactentes expostos ao HIV apresentaram maior escore bruto. Esse maior escore pode ser justificado pelo fato das famílias serem acompanhadas pela equipe do Senic/Craids, que procura garantir boa condição de saúde dessa família que se encontra em situação de vulnerabilidade. Portanto, é possível que desenvolvimento adequado desses lactentes seja reflexo 
dos cuidados e orientações passados às famílias acompanhadas.

Múltiplos fatores como ambientais, biológicos, sociais, econômicos e culturais influenciam o desenvolvimento neuropsicomotor. Assim, acompanhar o desenvolvimento neuromotor e ter parâmetros do padrão do desenvolvimento para lactentes expostos ao HIV é fundamental para identificar possíveis suspeitas ou atrasos do desenvolvimento neuromotor nessa população. Os lactentes expostos ao HIV apresentam o risco biológico eminente devido a exposição ao vírus, que pode afetar o desenvolvimento do $\mathrm{SNC}^{7,9,11,12,19}$. Além desse, há um risco adicional, a terapia antirretroviral, que também pode alterar o desenvolvimento do SNC e repercutir nas aquisições motoras desse lactente. A identificação precoce de suspeitas ou atrasos do desenvolvimento por meio de ferramentas e instrumentos adequados permite a intervenção imediata e amenizar possíveis sequelas de ordem motora.

Outro aspecto interessante em relação ao padrão de desenvolvimento dos lactentes expostos ao HIV refere-se às extremidades da curva percentílica do desenvolvimento, idades de RN a 3 meses e de 15 a 18 meses (Figura 1). Nestas idades, os pontos de corte são os mesmos independente da idade, indicando baixo poder de diferenciação da escala Motora Infantil de Alberta (AIMS) nessas idades ${ }^{26,28}$. Sacanni e Valentini ${ }^{26}$ afirmam que essa característica indica baixa capacidade de classificar os 
lactentes dessas faixas etárias entre típicas, suspeitas e com desenvolvimento atípico e que esse padrão deve-se ao pouco número de posições a serem avaliadas na faixa etária final da $\mathrm{AIMS}^{26}$. Dessa forma, deve-se ter maior cuidado ao utilizar AIMS nessas idades, e outros instrumentos podem ser utilizados, concomitantemente, para melhor avaliação do desenvolvimento do lactente.

A Escala Motora Infantil de Alberta foi construída no Canadá$^{24}$, e validada para a população brasileira ${ }^{26}$. Essa ferramenta possibilita identificar atrasos e anormalidades no desenvolvimento, permitindo, se necessário, intervir precocemente e minimizar os efeitos prejudiciais gerados. A escala vem sendo amplamente utilizada, em diferentes populações, por ser de fácil e rápida aplicação, possuir baixo custo de aquisição e utilização. Os resultados deste estudo demonstram a viabilidade da utilização da AIMS para avaliação dessa população específica.

O estudo pretendeu gerar subsídios para que profissionais da saúde possam avaliar e identificar o padrão de desenvolvimento motor de lactentes expostos ao HIV.

\section{CONCLUSÃO}

Lactentes expostos ao HIV possuem padrão de desenvolvimento neuromotor similar ao padrão de desenvolvimento neuromotor de lactentes não expostos ao HIV. Devido à similaridade entre as curvas de desenvolvimento, a avaliação do desenvolvimento dos lactentes brasileiros expostos ao HIV pode ser avaliado com 
base na curva de referência brasileira para lactentes, sem prejuízo na sua avaliação

\section{AGRADECIMENTOS}

Fonte financiadora: CNPq (Conselho Nacional de Desenvolvimento Científico e Tecnológico) bolsa de iniciação científica. Número de processo: 126026/20130

\section{REREFÊNCIAS}

1.Tani G, Meira Junior CM, Ugrinowitsch H, Benda RN, Chiviacowsky $S$, Corrêa UC. Pesquisa na área de comportamento motor: modelos teóricos, métodos de investigação, instrumentos de análise, desafios, tendências e perspectivas. Rev Educ Fís EUM 2010;21:1-52. http://dx.doi.org/10.4025/reveducfisv21n3p329-380

2.Soares DA, Van der Kamp J, Savelsbergh GJ, Tudella E. The effect of a short bout of practice on reaching behavior in late preterm infants at the onset of reaching: a randomized controlled trial. Res Dev Disabil 2013;34:4546-58.

http://dx.doi.org/10.1016/j.ridd.2013.09.028

3.Pedrosa C, Caçola P, Carvalhal MIMM. Fatores preditores do perfil sensorial de lactentes dos 4 aos 18 meses de idade. Rev Paul Pediatr 2015;33:160-6. http://dx.doi.org/10.1016/j.rpped.2014.11.016

4.Adolph KE, Franchak JM. The development of motor behavior. Wiley Interdiscip Rev Cogn Sci 2017;8:1-30.

http://dx.doi.org/10.1002/wcs.1430

5.Gonçalves GAC, Gonçalves AK, Perotti Júnior A. Desenvolvimento motor na teoria dos sistemas dinâmicos. Rev Motriz 1995;1:8-14.

6. Madaschi V, Paula CS. Medidas de avaliação do desenvolvimento infantil: uma revisão da literatura nos últimos cinco anos. Cad Pós Grad Dist Desenvol 2011;11:52-6.

7. Willrich A, Azevedo CCF, Fernandes JO. Desenvolvimento motor na infância: influência dos fatores de risco e programas de intervenção. Rev Neurocienc 2009;17:51-6.

8.Van Rie A, Harrington PR, Dow A, Robertson K. Neurologic and neurodevelopmental manifestations of pediatric HIV/AIDS: a global perspective. Eur J Paediatr Neurol 2007;11:1-9.

http://dx.doi.org/10.1016/j.ejpn.2006.10.006

9.Gómez C, Archila ME, Rugeles C, Carrizosa J, Rugeles MT, Cornejo JW. Estudio prospectivo comparativo sobre el desarrollo psicomotor de niños de madres positivas para el virus de inmunodeficiencia humana tipo 1 no infectados. Rev Neurol 2009;48:287-91. https://doi.org/10.33588/rn.4806.2008400 
10.Secretaria de Estado da Saúde de São Paulo. Centro de Referência e Treinamento em DST/AIDS - CRT-DST/AIDS-SP. Programa Estadual de DST/AIDS de São Paulo. Bol Epidemiol CRT-DST/AIDS $2011 ; 1: 1-112$.

11.Potterton J, Stewart A, Cooper P, Becker P. The effect of a basic home stimulation programme on the development of young children infected with HIV. Dev Med Child Neurol 2010;52:547-51. https://doi.org/10.1111/j.1469-8749.2009.03534.x

12.Walker SP, Wachs TD, Grantham-McGregor S, Black MM, Nelson $\mathrm{CA}$, Huffman $\mathrm{SL}$, et al. Inequality in early childhood: risk and protective factors for early child development. Lancet 2011;378:1325-38. https://doi.org/10.1016/S0140-6736(11)60555$\underline{2}$

13.Powis KM, Smeaton L, Ogwu A, Lockman S, Dryden-Peterson S, van Widenfelt $\mathrm{E}$, et al. Effects of in utero antiretroviral exposure on longitudinal growth of HIV-exposed uninfected infants in Botswana. J Acquir Immune Defic Syndr 2011;56:131-8.

https://doi.org/10.1097/QAI.0b013e3181ffa4f5

14. Resegue R, Puccini RF, da Silva EMK. Risk factors associated with developmental abnormalities among high-risk children attended at a multidisciplinary clinic. Sao Paulo Med J 2008;126:4-10.

http://dx.doi.org/10.1590/S1516-31802008000100002

15.Amorim RCA, Laurentino GEC, Barros KMFT, Ferreira ALPR, Moura Filho AG, Raposo MCF. Programa de saúde da família: proposta para identificação de fatores de risco para o desenvolvimento neuropsicomotor. Rev Bras Fisioter 2009;13:506-13. http://dx.doi.org/10.1590/S1413-35552009005000065

16.Santos DCC, Tolocka RE, Carvalho J, Heringer LRC, Almeira CM, Miquelote AF. Desempenho motor grosso e sua associação com fatores neonatais, familiares e de exposição à creche em crianças até três anos de idade. Rev Bras Fisioter 2009;13:173-9. http://dx.doi.org/10.1590/S1413-35552009005000025

17.Formiga CKMR, Linhares MBM. Avaliação do desenvolvimento inicial de crianças nascidas pré-termo. Rev Esc Enferm USP 2009;43:472-80. http://dx.doi.org/10.1590/S008062342009000200030

18.Souza ES, Magalhães LC. Desenvolvimento motor e funcional em crianças nascidas pré-termo e a termo: influência de fatores de risco biológico e ambiental. Rev Paul Pediatr 2012;30:462-70. http://dx.doi.org/10.1590/S0103-05822012000400002

19.Smith L, Adnams C, Eley B. Neurological and neurocognitive function of HIV - infected children commence on antiretroviral therapy. AS J of Child Healt 2008;2:108-13.

20.Recommendations of the US Public Health Service Task Force on the Use of Zidovudine to Reduce Perinatal Transmission of Human Immunodeficiency Virus. MMWR 1994;43:1-20. 
21.Ramos AD, Morais RLS. Vigilância do desenvolvimento neuropsicomotor de crianças de um programa DST/AIDS. Fisioter Pes 2011;18:371-6.

22. Marques HHS. Avaliação crítica dos efeitos adversos do tratamento anti-retroviral no feto, recém-nascido e lactente. Rev Bras Ginecol Obstet 2006;28:424-30. http://dx.doi.org/10.1590/S010072032006000700008

23. Foster CJ, Biggs RL, Melvin D, Walters MD, Tudor-Williams G, Lyall EGH. Neurodevelopmental outcomes in children with HIV infection under 3 years of age. Develop Med Child Neurol 2006;48:677-82. http://dx.doi.org/10.1017/S0012162206001423

24.Piper MC, Pinnell LE, Darrah J, Maguire T, Byrne PJ. Construction and validation of the Alberta Infant Motor Scale (AIMS). Can J Public Health 1992;83:46-50.

25. Bayley N. Bayley Scales of Infant Development III. San Antonio: The Psychological Corporation. 2006.

26.Saccani R, Valentini NC. Reference curves for the Brazilian Alberta Infant Motor Scale: percentiles for clinical description and follow-up over time. J Pediatr (Rio J) 2012;88:40-7. http://dx.doi.org/10.2223/JPED.2142

27.Sá CSC, Cardozo FN, Carvalho RP. Acompanhamento do desenvolvimento neuromotor de crianças expostas ao HIV. Temas Desenvol 2014;20:8-12.

28. Herrero D, Golçalves $H$, de Siqueira AAF, de Abreu LC. Escalas de desenvolvimento motor em lactentes: test of infant motor performance e a Alberta infant motor scale. Rev Bras Cresc Desenv Hum 2011;21:122-32.

29.Koenker RW, Basset GW. Regression quantiles. Econometr 1978;46:33-50.

30. Herrero D, Gallo PR, Fujimori M, Monteiro CBM, Valenti VE, Tavares CM, et al. Motor development of infants exposed to maternal human immunodeficiency virus (HIV) but not infected. Int Arch Med 2013;6:45. http://dx.doi.org/10.1186/1755-7682-6-45 